\title{
Utilização de Índices Radiomorfométricos em Exames de Imagem
} Use of radiomorphometric indices in imaging exams

\author{
Wilson Gustavo Cral" \\ Mariana Quirino Silveira* \\ Rosana Mara Adami Tucunduva** \\ Roberta Heiffig Handem Abujamra*** \\ Dagmar de Paula Queluz $z^{* * *}$
}

\section{Resumo}

A radiomorfometria consiste na aplicação de índices para avaliação da morfologia óssea em exames de imagem, principalmente em radiografias panorâmicas. Objetivo: o objetivo deste estudo é analisar as principais indicações dos índices radiomorfométricos em estudos publicados na literatura científica. Métodos: uma busca textual foi realizada utilizando a palavra-chave "radiomorphometric indices" na base de dados PubMed, procurando responder à seguinte questão: Qual a aplicação dos índices radiomorfométricos em exames de imagem? Resultados: 36 artigos sobre esse tópico foram identificados entre os anos de 1999 a 2016, sendo o periódico Dentomaxillofacial Radiology o que possui a maior quantidade de estudos $(25,0 \%)$. Conclusão: a principal utilização dos índices radiomorfométricos inclui a avaliação da densidade óssea de diferentes grupos de pacientes, podendo não apresentar qualquer alteração sistêmica ou possuir doenças, como osteoporose e osteogênese imperfeita. As vantagens dos índices radiomorfométricos incluem principalmente acessibilidade e baixo custo, porém requerem conhecimento e treinamento técnico.

Palavras-chave: Densitometria. Radiografia Panorâmica. Densidade óssea.

\section{Introdução}

A radiomorfometria consiste na aplicação de índices para avaliação da morfologia óssea em exames de imagem. Os índices radiomorfométricos são predominantemente fundamentados em medidas de osso cortical, por ser este mais facilmente visualizado em radiografias do que o osso trabecular ${ }^{1}$.

A utilização dos índices radiomorfométricos, em sua maioria, é aplicada em radiografias panorâmicas, uma vez que a maior parte da população é submetida a este exame, principalmente em estudos científicos de densidade óssea em indivíduos com osteoporose $^{2}$. A primeira associação entre a osteoporose e a perda óssea na mandíbula e na maxila de seres humanos foi realizada por Groen et al. ${ }^{3}$, em 1960. Segundo estudos, a espessura da cortical inferior da mandíbula é reduzida em pacientes com essa doença ${ }^{3}$.

Atualmente, são utilizadas várias técnicas para diagnosticar a osteoporose. A técnica padrão-ouro é a densitometria óssea usando a técnica DXA (Dual Energy Xray Absorptiometry). A falta de acesso da população ao exame é um fator que dificulta sua utilização como método de rastreamento popula-

*** Doutora em Ciências Odontológicas, Estomatologia, Faculdade de Odontologia de Bauru, Universidade de São Paulo (USP), Bauru, SP, Brasil.

Professora associada do Departamento de Odontologia Social da Faculdade de Odontologia de Piracicaba, Universidade Estadual de Campinas (UNICAMP), Piracicaba, SP, Brasil. 
cional $^{4}$. Considerando, portanto, a dificuldade destes indivíduos para a realização da densitometria óssea ${ }^{5}$, esforços devem ser despendidos visando a maximizar a utilização adequada dos densitômetros existentes, por exemplo, identificando melhor a parcela da população com maior risco de baixa densidade mineral óssea, otimizando a relação custo-benefício.

O objetivo deste trabalho é analisar as principais utilizações dos índices radiomorfométricos em estudos publicados na literatura científica, discuti-los segundo as suas principais aplicações e importância, além de categorizá-los, de acordo com o periódico e ano de publicação.

\section{Metodologia}

Para a elaboração da revisão de literatura as seguintes etapas foram percorridas: definição da questão norteadora e objetivos da pesquisa, busca na literatura; análise e categorização dos estudos, apresentação e discussão dos resultados. Para guiar a pesquisa na base de dados, formulou-se a seguinte questão: Qual a aplicação dos índices radiomorfométricos em exames de imagem? Foi utilizada a palavra-chave "radiomorphometric indices" na plataforma PubMed.

Definições dos principais índices:

a) Índice cortical mandibular (MCI): Qualitativo e visual, avalia o grau de reabsorção da cortical inferior da base da mandíbula em: C1 a margem da cortical está clara e nítida em ambos os lados; C2 - a superfície endosteal apresenta defeitos semilunares (reabsorções lacunares) ou a superfície apresenta resíduos de cortical; C3 - a camada cortical está extremamente porosa ${ }^{6}$.

b) Índice antegoníaco (AI): Consiste na aplicação de uma linha "A" paralela à base da mandíbula; uma segunda linha " $\mathrm{B}$ " tangente à porção anterior do ramo da mandíbula (processo coronóide); e uma linha " $\mathrm{C}$ " perpendicular à linha "A" no ponto de cruzamento com a linha "B", onde se mede a espessura da cortical da base da mandíbula ${ }^{6}$.

c) Índice mentoniano (MI): É a medida da largura da região cortical do forame mentoniano. Uma linha paralela ao eixo longo da mandíbula e tangencial à borda inferior da mandíbula é traçada; e uma linha perpendicular a esta tangente, que cruza a borda inferior do forame mentoniano ${ }^{6}$.

d) Índice mandibular panorâmico (PMI): é a medida da distância entre a margem inferior do forame mentoniano e cortical inferior da mandíbula ${ }^{6}$.

\section{Resulltados}

\section{Distribuicção dos artigos}

Foram encontrados 36 estudos, sendo 34 pesquisas científicas e 2 revisões de literatura.

Tabela 1 -Distribuição dos artigos, segundo os periódicos, em dezembro de 2016

\begin{tabular}{l|c|l|c}
\hline \multicolumn{1}{c|}{ Periódico } & № artigos & & Ano \\
\hline Dentomaxillofac Radiol. & 9 & $1999-2001-2008-2010-2010-2013-2015-2016-2016$ & $25,0 \%$ \\
J Clin Diagn Res. & 2 & $2014-2015$ & $5,55 \%$ \\
Osteoporos Int. & 2 & $2002-2012$ & $5,55 \%$ \\
Oral Surg Oral Med Oral Pathol Oral Radiol Endod. & 2 & $2005-2010$ & $5,55 \%$ \\
Arch Oral Biol. & 1 & 2015 & $2,7 \%$ \\
J Midlife Health. & 1 & 2015 & $2,7 \%$ \\
Open Dent J. & 1 & 2015 & $2,7 \%$ \\
Hippokratia & 1 & 2014 & $2,7 \%$ \\
J Contemp Dent Pract. & 1 & 2014 & $2,7 \%$ \\
Skeletal Radiol. & 1 & 2014 & $2,7 \%$ \\
Am J Med Sci. & 1 & 2013 & $2,7 \%$ \\
Oral Health Dent Manag. & 1 & 2013 & $2,7 \%$ \\
Gerodontology & 1 & 2012 & $2,7 \%$ \\
Morphol & 1 & 2011 & $2,7 \%$ \\
Iran J Radiol. & 1 & 2011 & $2,7 \%$ \\
ISRN Rheumatol. & 1 & 2011 & $2,7 \%$ \\
Med Oral Patol Oral Cir Bucal & 1 & 2011 & $2,7 \%$ \\
J Dent (Tehran) & 1 & 2010 & $2,7 \%$ \\
J Indian Soc Periodontol. & 1 & 2010 & $2,7 \%$ \\
Medicina (Kaunas) & 1 & 2010 & $2,7 \%$ \\
J Prosthet Dent. & 1 & 2009 & $2,7 \%$ \\
Joint Bone Spine & 1 & 2009 & $2,7 \%$ \\
Int Dent J. & 1 & 2007 & $2,7 \%$ \\
Coll Antropol. & 1 & 2002 & $2,7 \%$ \\
J Gerontol A Biol Sci Med Sci. & 1 & 2002 & $2,7 \%$ \\
\hline
\end{tabular}


Ao analisar o tipo de publicação, verificou-se que $36(100 \%)$ eram artigos científicos. A maior parte deles, $25 \%$, foram estudos publicados na Revista Dentomaxillofacial Radiology, sendo também pioneira em trabalhos de índices radiomorfométricos, em 1999.

Em relação aos anos das publicações, verificou-se que em 1999, 2001, 2005, 2007 e 2008 houve um trabalho publicado em cada um deles, somando 13,5\% das publicações. Os anos de 2009, 2012 e 2016 seguiram com dois trabalhos publicados ao ano, totalizando 16,5\% das publicações. Nos anos de 2002 e 2013 foram encontrados três trabalhos ao ano, ou $8,33 \%$. Em 2011 e 2014 foram encontradas quatro publicações ao ano (11,11\%), e em 2015 obteve-se a maior quantidade de trabalhos publicados, sendo cinco publicações, ou 13,8\%. Os dados encontram-se na Tabela 1.

Ao analisar as principais aplicações dos índices radiomorfométricos, observa-se que a maior parte dos estudos avaliaram pacientes em condições sistêmicas normais (25\%), com o objetivo de estudar a densidade óssea de indivíduos de diferentes populações, gêneros e idades. A doença sistêmica com maior número de publicações foi a osteoporose (25\%).

Tabela 2 - Principais aplicações dos índices radiomorfométricos, segundo o perfil da amostra dos trabalhos científicos

\begin{tabular}{l|c|c}
\hline \multicolumn{1}{c|}{ Aplicação dos índices } & № artigos & $\%$ \\
\hline Condição sistêmica normal & 16 & $44,4 \%$ \\
Osteoporose & 9 & $25,0 \%$ \\
Mulher pós-menopausa & 4 & $11,1 \%$ \\
Edentulismo & 2 & $5,55 \%$ \\
Revisão de literatura & 2 & $5,55 \%$ \\
Doença falciforme & 1 & $2,7 \%$ \\
Doença periodontal & 1 & $2,7 \%$ \\
Osteogênese imperfeita & 1 & $2,7 \%$ \\
\hline
\end{tabular}

\section{Discussão}

Os índices radiomorfométricos possuem a capacidade de predizer o diagnóstico de baixa densidade mineral óssea e de osteoporose, sendo esta condição um grande alvo dos trabalhos científicos sobre este tema. Novos estudos são necessários para verificar se esses índices podem também predizer o risco de fraturas por osteoporose e elucidar se, em homens idosos, os índices radiomorfométricos possuem a mesma aplicabilidade que a observada em mulheres na pós-menopausa ${ }^{7}$.

O periódico Dentomaxillofacial Radiology foi precursor no estudo de índices radiomorfométricos, possuindo também a maior quantidade de trabalhos publicados (25\%), de 1999 a 2016.

Os índices radiomorfométricos, como o índice cortical mandibular (MCI), índice mandibular panorâmico (PMI), índice mentoniano (MI), índice antegoníaco (AI) e índice goníaco (GI) foram desen- volvidos para avaliar e quantificar a qualidade da massa óssea mandibular. O índice cortical mandibular, por exemplo, é um índice qualitativo, que depende apenas da análise visual da radiografia panorâmica, apresentando a capacidade de predizer baixa densidade mineral ${ }^{6}$. Para isso, considera-se importante a padronização dos exames, através do ajuste de quilovoltagem (KV) e miliampere mAs segundo, dos aparelhos. O diagnóstico dessas alterações é promissor através da Tomografia Computadorizada de Feixe Cônico, visto que na literatura ainda há poucos trabalhos publicados.

No estudo de Bajoria $^{8}$ (2015) foram determinados os índices radiomorfométricos de radiografias panorâmicas digitais de 23 pacientes acima de 25 anos, com ausência de qualquer patologia que comprometa o metabolismo ósseo e, pelo menos, um forame mentoniano evidente na radiografia. Os autores relataram que a avaliação de todos os índices foi possível nas radiografias panorâmicas, havendo diferença estatisticamente significativa entre os grupos mais jovens e mais velhos. Os índices apresentaram valores menores para o gênero feminino em relação ao masculino de mesma faixa etária ${ }^{8}$.

Em relação à população brasileira, é interessante observar a conclusão do estudo de Alonso et al. ${ }^{9}$ (2011), confirmando a existência de diferenças significativas em relação à idade e qualidade óssea entre os gêneros, sendo as mulheres com índices de densidade óssea menores comparadas ao gênero masculino. Os resultados confirmam significativamente que as alterações relacionadas à idade e sexo de um paciente interferem na qualidade óssea de forma importante ${ }^{9}$.

A osteoporose é uma condição prevalente no mundo todo, sendo muito comum nos idosos, especialmente em mulheres pós-menopausa. É um distúrbio que está associado a uma diminuição da densidade da massa óssea, podendo resultar, principalmente, em fraturas ósseas nos indivíduos afetados ${ }^{10}$. Khatoonabad et al. ${ }^{11}$ (2011), relacionaram os índices radiomorfométricos com a densitometria óssea e marcadores bioquímicos do quadril de mulheres pós-menopausa. Os índices utilizados foram o índice cortical mandibular, o índice mandibular panorâmico e a razão de reabsorção da crista alveolar. Os autores estudaram 140 mulheres, com idade entre 44 e 82 anos, que não haviam experimentado a menstruação no último ano. Não foi encontrada relação dos índices radiomorfométricos com biomarcadores do turnover ósseo do quadril, sendo, para isso, necessários estudos adicionais ${ }^{11}$.

Alguns estudos têm apontado a relação entre esses índices e a densitometria óssea, porém, encontrando resultados contraditórios. Marandi et al. ${ }^{12}$ (2010), também com o objetivo de avaliar a possibilidade de utilização destes parâmetros como indicadores de osteoporose, realizaram um estudo analítico transversal para avaliar a eficácia diagnóstica 
dos índices panorâmicos da mandíbula (Índice mentoniano, índice cortical mandibular e índice mandibular panorâmico) e determinar sua correlação com a densidade mineral óssea do colo femoral e das vértebras lombares (L2-L4). Os índices mandibulares de 67 mulheres com mais de 35 anos foram medidos a partir de radiografias panorâmicas, sendo realizada densitometria óssea no colo femoral e nas vértebras lombares (L2-L4), utilizando a técnica DXA (Dual Energy X-ray Absorptiometry). Embora encontrados resultados estatisticamente significantes nos índices cortical mandibular e mentoniano, foi concluído que os índices radiomorfométricos utilizadas na mandíbula não foram altamente suficientes para uma avaliação precisa ${ }^{12}$.

A comparação do uso de índices radiomorfométricos entre a técnica DXA e a Tomografia Computadorizada de Feixe Cônico (TCFC) de mandíbula foram realizadas no estudo de Mostafa et al. ${ }^{13}$ (2016), em pacientes do gênero feminino, pós-menopausa, com idades entre 55 e 70 anos, divididas em dois grupos: osteoporóticas e grupo controle. Foi concluído que os resultados dos índices do grupo controle foram superiores às mulheres osteoporóticas e que os índices radiomorfométricos da TCFC poderiam ser usados como uma ferramenta adjuvante para encaminhar pacientes com risco de osteoporose para avaliação adicional, mostrando-se um exame de imagem promissor na detecção dos pacientes de risco e futuras pesquisas envolvendo a radiomorfometria $^{13}$.

A relação entre doença periodontal e osteoporose também foi investigada usando índices radiomorfométricos. Moeintaghavi et al. ${ }^{14}$ (2014), estudaram a relação entre doença periodontal e osteoporose através de índices radiomorfométricos, utilizando radiografias panorâmicas de pacientes com periodontite crônica e indivíduos saudáveis. A análise foi realizada através dos seguintes índices: índice cortical mandibular (MCI) e índice mentoniano (MI). Entre os pacientes, 58,6\% eram do gênero masculino e $41,4 \%$ do gênero feminino e o único índice estatisticamente significante foi o MCI $(p=0,028)$. Apesar disso, o estudo não mostrou uma forte evidência entre doença periodontal e osteoporose ${ }^{14}$.

Procurando verificar a relação dos índices radiomorfométricos em radiografias panorâmicas de crianças com diferentes tipos de osteogênese imperfeita, em tratamento com pamidronato intravenoso, conclui-se que, para este grupo de risco, as crianças apresentam corticais mandibulares mais finas e porosas no início do tratamento, e que a melhoria da qualidade do osso cortical pós-tratamento pode ocorrer conforme o tipo de osteogênese imperfeita e número de ciclos de pamidronato. A análise da densidade óssea torna-se de extrema importância para a avaliação do progresso da utilização de pamidronato intravenoso ${ }^{15}$.

Novos estudos são necessários para verificar se esses índices podem também predizer o risco de fra- turas por osteoporose e elucidar se, em homens idosos, os índices radiomorfométricos possuem a mesma aplicabilidade que a observada em mulheres na pós-menopausa ${ }^{7}$.

\section{Conclusões}

Embora a densitometria óssea seja o exame padrão-ouro para avaliação da densidade óssea, a radiografia panorâmica pode ser um indicador dessas alterações na mandíbula, contribuindo na identificação de indivíduos com baixa densidade óssea não diagnosticada ou com osteoporose, principalmente, e sugerir uma maior investigação, permitindo interceptar o progresso da doença. Portanto, a aplicação dos índices radiomorfométricos inclui a avaliação da densidade óssea de diferentes grupos de pacientes, podendo ou não apresentar alterações sistêmicas.

Estudos radiomorfométricos podem ser usados como complemento ao questionário anamnésico dos indivíduos do gênero feminino na faixa etária de 45-60 anos, uma vez que esta faixa etária apresenta maior risco de alterações do metabolismo ósseo. Novos estudos são bem-vindos para avaliar se a os índices radiomorfométricos são capazes de predizer o risco de fraturas e elucidar se, em homens idosos, possuem a mesma aplicabilidade que a observada em mulheres na pós-menopausa.

\section{Abstract}

Radiomorphometry consists in the application of indices for assessing bone morphology in imaging tests, mainly in panoramic radiographs. Objective: this study aimed to analyze the main indications of radiomorphometric indices in studies published in the scientific literature. Methods: a text search was performed using the keyword "radiomorphometric indices" in the PubMed database, attempting to answer the following question: What is the application of radiomorphometric indices on imaging tests? Results: 36 articles on this topic were identified between the years of 1999 and 2016, and the Dentomaxillofacial Radiology journal showed the highest number of studies (25.0\%). Conclusion: the main use of radiomorphometric indices includes the assessment of bone density of different groups of patients and may not present any systemic changes or indicate diseases such as osteoporosis and osteogenesis imperfecta. The advantages of radiomorphometric indices mainly include accessibility and low cost, but they require knowledge and technical training.

Keywords: Densitometry. Panoramic radiograph. Bone density. 


\section{Referências}

1. Bollen AM, Taghuci A, Hujoel PP, Hollender LG. Case-control study on self reported osteoporotic fractures and mandibular cortical bone. Oral Surg Oral Med Oral Pathol Oral Radiol Endod 2000; 90(4):518-24.

2. Taguchui A, Suei Y, Ohtsuka M, Otani K, Tanimoto K, Ohtaki M. Usefulness of panoramic radiography in the diagnosis of postmenopausal osteoporosis in women. Width and morphology of inferior cortex of the mandible. Dentomaxillofac Radiol 1996; 25(5):263-7.

3. Groen JJ, Duyvensz F, Halsted JA. Diffuse alveolar atrophy of the jaw (non-inflammatory form of paradental disease) and presenile osteoporosis. Gerontol Clin 1960; 2:68-86.

4. Costa-Paiva L, Horovitz AP, Santos AO, Fonsechi-Carvasan GA, Pinto-Neto AM. Prevalência da osteoporose em mulheres na pós-menopausa e associação com fatores clínicos e reprodutivos. RBGO 2003; 25(7):507-12.

5. Kanis JA, Johnell O. Requirements for DXA for the management of osteoporosis in Europe. Osteoporos Int 2005; 16(3):229-38.

6. Leite AF, Figueiredo PTS, Guia CM, Melo NS, Paula AP. Radiografia panorâmica - Instrumento auxiliar no diagnóstico da osteoporose. Rev Bras Reumatol 2008; 48(4):226-33.

7. Cova DMP, Panzarella FK, Montebelo Filho A, Tavano O, Junqueira JLC, Oliveira LB. Estudo radiomorfométrico com indicador de risco de osteoporose. Pesq Bras Odontoped Clin Integr 2012; 12(2):217-22.

8. Bajoria AA. Evaluation of Radiomorphometric Indices in Panoramic Radiograph - A Screening Tool. Open Dent 2015; 9:303-10.

9. Alonso MB, Cortes AR, Camargo AJ, Arita ES, Haiter-Neto F, Watanabe PC. Assessment of panoramic radiomorphometric indices of the mandible in a brazilian population. ISRN Rheumatol. 2011; 2011:854287. doi:10.5402/2011/854287

10. Leite AF, Figueiredo PTS, Guia CM, Melo NS, de Paula AP. Correlations between seven panoramic radiomorphometric indices and bone mineral density in postmenopausal women. Oral Surg Oral Med Oral Pathol Oral Radiol Endod 2010; 109(3):449-56.

11. Khatoonabad M, Aghamohammadzade N, Taghilu H, Esmaeili F, Khamnei HJ. Relationship Among Panoramic Radiography Findings, Biochemical Markers of Bone Turnover and Hip BMD in the Diagnosis of Postmenopausal Osteoporosis. Iran J Radiol 2011; 8(1):23-8.

12. Marandi S, Bagherpour A, Imanimoghaddam M, Hatef M, Haghighi A. Panoramic-based mandibular indices and bone mineral density of femoral neck and lumbar vertebrae in women. J Dent (Tehran) 2010; 7(2):98-106.

13. Mostafa RA, Arnout EA, Abo El-Fotouh MM. Feasibility of cone beam computed tomography radiomorphometric analysis and fractal dimension in assessment of postmenopausal osteoporosis in correlation with dual X-ray absorptiometry. Dentomaxillofac Radiol. 2016; 45(7):20160212. doi: 10.1259/ dmfr.20160212.

14. Moeintaghavi A, Hosseinuiarch H, Tabassi SM. The comparison of mandibular radiomorphometric indices in panoramic radiography between patients with chronic periodontitis and healthy individuals. J Contemp Dent Pract. 2014 Jul; 15(4):461-5.

15. Apolinario AC, Sindeaux R, Souza Figueiredo PT, Guimarães AT, Acevedo AC, Castro LC et al. Dental panoramic indices and fractal dimension measurements in osteogenesis imperfecta children under pamidronate treatment. Dentomaxillofac Radiol. 2016; 45(4):20150400. doi:10.1259/ dmfr.20150400.
Endereço para correspondência:

Wilson Gustavo Cral

Faculdade de Odontologia de Bauru,

Universidade de São Paulo

Departamento de Estomatologia

Al. Octávio Pinheiro Brisola, 9-75

CEP-17012-901 Cidade Universitária

Bauru - São Paulo, BRASIL

E-mail:wgcral@gmail.com

Recebido: 18/11/2016. Aceito: 03/02/2017. 